\title{
IN THIS ISSUE - FeBRUARY 1, 2007
}

\section{JAVMA News}

Issues in state public policy were the subject of a recent AVMA symposium, while the national spotlight turned to the issue of the safety of food from animal clones.

See PAGE 322

\section{Letters to the Editor}

See PAGE 338

FOOD SUPPLY VETERINARY MEDICINE

\section{The need for a food supply-exclusive college of veterinary medicine}

The pressing need for more graduates who will enter the field of food supply veterinary medicine has been made abundantly clear. The author argues that the time has come to establish a new veterinary medical institution with the express purpose of training veterinarians for careers in food supply veterinary medicine.

See PAGE 342

\section{What Is Your Diagnosis?}

See PAGES 345, 347



VETERINARY MEDICINE AND THE LAW

\section{Veterinary legal issues: 2006 in review}

As pet owners continue to embrace the human-animal bond and society struggles with the impact of that bond on the legal status of animals, the importance of animal law continues to grow. The past year in particular has been a busy time in the field of animal law.
ORIGINAL STUDY

\section{Limb function following laser or scalpel onychectomy in cats}

Previous authors have suggested that laser onychectomy is associated with reduced postoperative pain, compared with other forms of onychectomy, but such claims appear to be largely anecdotal. In a study of 20 healthy cats randomly assigned to undergo unilateral onychectomy with a $\mathrm{CO}_{2}$ laser or scalpel, cats that underwent laser onychectomy were found to have significantly higher ground reaction forces and a significantly higher ratio for peak vertical force on the operated limb versus all other limbs 1, 2, and 12 days after surgery. None of the cats required rescue analgesia.

See PAGE 353

\section{ORIGINAL STUDY \\ Effect of vaccination on parvovirus antigen testing in kittens}

Current guidelines for shelters suggest that all cats be inoculated with a modified-live feline panleukopenia virus vaccine on admission. However, vaccination may sometimes result in transient fecal shedding of viral antigens that cannot be distinguished from natural infection with currently available diagnostic test kits. In a study of 64 kittens inoculated with 1 of 8 commercial multivalent vaccines, $13(20 \%)$ had at least 1 positive result after vaccination when feces were tested for parvovirus antigen. Nevertheless, the benefits associated with universal vaccination against FPV for cats in high-risk environments appeared to outweigh the negative impact on diagnostic test accuracy.

See PAGE 359

\section{ORIGINAL STUDY}

\section{Reproducibility and accuracy of devices used to measure urine $\mathrm{pH}$ in dogs}

Reagent strips are commonly used in veterinary practice to measure urine $\mathrm{pH}$, but may not be sufficiently accurate. A study of 201 free-catch urine samples from 114 dogs revealed that 3 of 4 portable $\mathrm{pH}$ meters yielded measurements that closely approximated those obtained with a reference benchtop $\mathrm{pH}$ meter. However, pH values obtained with reagent strips or $\mathrm{pH}$ paper had only moderate or poor agreement with reference values. Results suggest that the accuracy of reagent strips is acceptable when estimation of $\mathrm{pH}$ is sufficient, but that urine $\mathrm{pH}$ should be determined with a portable or benchtop pH meter when accurate measurements are crucial. See PAGE 364

CLINICAL REPORT

Treatment of traumatic cervical myelopathy in a dog

A 9-year-old dog was evaluated for cervical myelopathy after a traumatic incident that resulted in subluxation of $\mathrm{C} 2$ and C3, 


\section{FEBRUARY 1, 2007-IN ThIS ISSUE}

with dorsal displacement of C3. On admission, the dog could not ventilate spontaneously and was tetraplegic. The subluxation was stabilized with pins, screws, and polymethyl methacrylate. Positive-

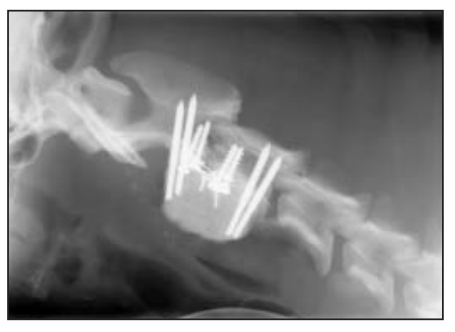
pressure ventilation was

provided for 37 days, along with extensive physical therapy. The dog was discharged 46 days after injury. At the time of discharge, the dog could walk, although it had severe ataxia and tetraparesis. One year later, even though moderate ataxia and tetraparesis persisted, the dog was able to run.

See PAGE 370

\section{CLINICAL REPORT}

\section{Subtotal penile amputation and preputial urethrostomy in a dog}

A 6-year-old dog was evaluated because of intermittent bleeding during urination. Strangulation of the penis secondary to paraphimosis with necrosis of the cranial portion of the penile shaft was diagnosed. A midline preputiotomy was performed to expose the penile shaft, and amputation was performed caudal to the preputial fornix. The terminal portion of the urethra was anastomosed to the preputial mucosa, allowing the dog to urinate through the preputial orifice and decreasing the potential for local skin irritation during urination.

See PAGE 375

ORIGINAL STUDY

\section{Ultrasound-assisted collection of CSF from the lumbosacral space in equids}

Abnormal osseous conformation, heavy musculature, and fat deposition in the lumbosacral region may make collection of CSF from the lumbosacral space more difficult in equids, increasing the risk of trauma and of contamination with blood or tissue. Cerebrospinal fluid was successfully obtained from 35 of 37 horses following puncture along the midline just cranial to the cranial borders of the most superficial portions of the tuber sacrale, as determined by ultrasonography. Ultrasonography was a useful aid in the collection of CSF from the lumbosacral space in these horses and appeared to decrease the risk of trauma and contamination.

See PAGE 378

ORIGINAL STUDY

\section{Human-directed aggression in miniature pet pigs}

Because swine social behavior differs from that of other domesticated animals, it is not feasible to extrapolate from what is known about human-directed aggression in dogs, cats, and horses and apply it to miniature pigs kept as pets. An Internet-based survey of 222 owners of miniature pet pigs revealed that 142 (64\%) owners had experienced at least 1 episode of human-directed aggression and 69 (31\%) experienced such episodes at least once a month. Sex, age at weaning, age at neutering, and environmental enrichment were not associated with the incidence of human-directed aggression, but the presence of a conspecific was associated with a decreased likelihood of aggression.

See PAGE 385

ORIGINAL STUDY

\section{Population pharmacokinetics of orally administered marbofloxacin in juvenile harbor seals}

Collection of blood samples for pharmacokinetic studies in freeranging or captive wildlife is challenging because of the difficulties in restraining such animals and the stress associated with restraint. Therefore, population pharmacokinetic analyses are a potentially useful approach for obtaining data from a population of wild animals. Analysis of blood samples from 55 juvenile harbor seals being treated with marbofloxacin $1 \leq 3$ samples/seal) for various conditions revealed that a dosage of $5 \mathrm{mg} / \mathrm{kg}$ (2.3 mg/lb), PO, every 24 hours should be efficacious for treatment of infections caused by susceptible bacteria other than Pseudomonas aeruginosa.

See PAGE 390 